\title{
A dynamic Tresca's frictional contact problem with damage for thermo elastic-viscoplastic bodies
}

\author{
Ilyas Boukaroura and Seddik Djabi
}

\begin{abstract}
We consider a dynamic contact problem between an elastic-viscoplastic body and a rigid obstacle. The contact is frictional and bilateral, the friction is modeled with Tresca's law with heat exchange. We employ the elastic-viscoplastic with damage constitutive law for the material. The evolution of the damage is described by an inclusion of parabolic type. We establish a variational formulation for the model and we prove the existence of a unique weak solution to the problem. The proof is based on a classical existence and uniqueness result on parabolic inéqualities, differentiel equations and fixed point argument.
\end{abstract}

Mathematics Subject Classification (2010): 74M10, 74M15, 74F05, 74R05, 74C10.

Keywords: elastic-viscoplastic, temperature, variational inequality, fixed point.

\section{Introduction}

The modelization of a contact phenomenon is determined by a set of assumptions influencing on the form and structure of partial differential equations system or on boundary conditions of the associated mathematical model.

Among the assumptions influencing the partial differential equations system: Hypothesis about the geometry of the deformation (small deformation or others), Hypothesis about the mechanical process (quasi-static or dynamic), Hypothesis about the laws of material behavior (elastic, viscoelastic,...).

The model equations can be influenced by additional phenomena (thermal, piezoelectric,...).

The boundary conditions on the contact surface are described in both normal direction and in the tangential plane, these are called boundary conditions of friction.

In the direction of normal, we have unilateral and bilateral contact (when there is no separation between the body and the obstacle). The normal compliance (when the obstacle is deformable). 
The boundary conditions are also influenced by several phenomena accompanying the contact with friction, such as adhesion, wear, thermal effects, friction threshold dependence with respect to sliding or the sliding speed.

The contact between deformable bodies are very common in the industry and everyday life, contact of braking pads with wheels, tires with roads, pistons with skirts or the complex metal.

Recently we investigated a number of problems related to quasistatic contact for thermo mechancical models coupled or uncoupled. In particular, models uncoupled thermo viscoplastic were considered in [10]. In this case the consitutive equation law depends on two parameters $\theta, \chi$, where $\theta$ be interpreted as absolute temperature.

Different models have been developed to describe the interaction between the thermal and mechanical field see $[3,11]$. A thermo elastic-viscoplastic body is considered in $[6,11]$.

Initial and boundary value problems for termo mechanical models were studied by many authors. So, existence and uniqueness result concerning the uncoupled thermo viscoelastic was obtained in [10] using a monotony method.

A quasistatic contact problem with friction and adhesion has been analized in [12] for viscoelastic body with long memory. The constitutive laws with internal states variables has been used in various publications see for example $[4,5,7]$.

The damage is one of the internal state variable considered by many authors, we can see $[1,3,6,9]$.

In this paper we consider the processes frictional contact between a termo elastic viscoplastic body with damage. We assume that the process is dynamic.

This article is organized as follows. In Section 2 we describe the mathematical model for the problem. In Section 3 we introduce some notation, list the assumptions on the problem's data, and derive the variational formulation of the model. Finally in Section 4 we state our main existence and uniqueness result which is based on classical result of nonlinear first order evolution inequalities, equations with monotone operators and the fixed point arguments.

For the mathematical problem we consider a rate-type constitutive equation for bodies of the form

$$
\boldsymbol{\sigma}=\mathcal{A} \varepsilon(\dot{\mathbf{u}})+\mathcal{G}(\varepsilon(\mathbf{u}), \xi)+\int_{0}^{t} \mathcal{F}(\boldsymbol{\sigma}(s)-\mathcal{A} \varepsilon(\dot{\mathbf{u}}(s)), \varepsilon(\mathbf{u}(s))) d s-C_{e} \theta,
$$

in which:

$\mathbf{u}, \boldsymbol{\sigma}$ represent, respectively, the displacement field and the stress field where the dot above denotes the derivative with respect to the time variable;

$\xi, \theta$ represent the damage, and the temperature;

$\mathcal{A}, \mathcal{G}$ and $\mathcal{F}$ are, respectively, nonlinear operators describing the purely viscous, the elastic and the viscoplastic properties of the material;

$C_{e}=\left(c_{i j}\right)$ represents the thermal expansion tensor.

The differential inclusion used for the evolution of the damage field is

$$
\dot{\xi}-k_{1} \Delta \xi+\partial \varphi_{F}(\xi) \ni S(\varepsilon(\mathbf{u}), \xi), \quad \text { in } \Omega \times(0, T),
$$


where $\varphi_{F}(\xi)$ denotes the subdifferential of the indicator function of the set $F$ of admissible damage functions defined by

$$
F=\left\{\xi \in H^{1}(\Omega) ; 0 \leq \xi \leq 1, \text { a.e.in } \Omega\right\}
$$

and $S$ are given constitutive functions which describe the sources of the damage in the system. When $\xi=0$ the material is completely damaged, when $\xi=1$ the material is undamaged, and for $0<\xi<1$ there is partial damage.

The evolution of the temperature field $\theta$ is governed by the heat equation, obtained from the conservation of energy and defined by the following differential equation for the temperature

$$
\dot{\theta}-\operatorname{div} K(\Delta \theta)=r(\dot{\mathbf{u}}, \xi)+\mathbf{q}
$$

$K$ represent the thermal conductivity tensor, $q(t)$ represent the density of volume heat source and $r$ is non linear function of velocity.

\section{Problem statement}

We consider an elasto-viscoplastic body which occupies a bounded domain $\Omega$ of the space $\mathbb{R}^{d}(d=2,3)$. For $\Omega$, the boundary $\Gamma$ is assumed to be Lipschitz continuous, and is partitioned into three disjoint measurable parts $\Gamma_{1}, \Gamma_{2}$ and $\Gamma_{3}$, such that meas $\Gamma_{1}>0$. Let $T>0$ and let $[0, T]$ denotes the time interval of interest. The body $\Omega$ is clamped on $\Gamma_{1} \times(0, T)$, and therfore, the displacement field vanishes there. Surface traction of density $\mathbf{f}_{2}$ act on $\Gamma_{2} \times(0, T)$ and a body force of density $\mathbf{f}_{0}$ acts on $\Omega \times(0, T)$. Morever the process is dynamic, and thus the inertial terms are included in the equation of motion. The material is assumed to behave according to the general elasto-viscoplastic constitutive law with damage and thermal effects given by (1.1)

With the assumption above, the classical formulation of a dynamic contact between an elasto-viscoplastic body and an obstacle with damage and thermal effects is the following.

Problem P. Find a displacement field $\mathbf{u}: \Omega \times(0, T) \rightarrow \mathbb{R}^{d}$, a stress field $\boldsymbol{\sigma}: \Omega \times$ $(0, T) \rightarrow \mathbb{S}^{d}$, a temperature $\theta: \Omega \times(0, T) \rightarrow \mathbb{R}$, and the damage field $\xi: \Omega \times[0, T] \rightarrow \mathbb{R}$ such that

$$
\begin{gathered}
\boldsymbol{\sigma}=\mathcal{A} \varepsilon(\dot{\mathbf{u}})+\mathcal{G}(\varepsilon(\mathbf{u}), \xi)+\int_{0}^{t} \mathcal{F}(\boldsymbol{\sigma}(s)-\mathcal{A} \varepsilon(\dot{\mathbf{u}}(s)), \boldsymbol{\varepsilon}(\mathbf{u}(s))) d s-C_{e} \theta \\
\dot{\theta}-\operatorname{div} K(\Delta \theta)=r(\dot{\mathbf{u}}, \xi)+\mathbf{q}, \quad \text { on } \Omega \times(0, T), \\
\dot{\xi}-k_{1} \Delta \xi+\partial \varphi_{F}(\xi) \ni S(\varepsilon(\mathbf{u}), \xi), \quad \text { in } \Omega \times(0, T), \\
\operatorname{div} \boldsymbol{\sigma}+\mathbf{f}_{0}=\rho \ddot{\mathbf{u}} \quad \text { in } \Omega \times(0, T), \\
\mathbf{u}=0 \quad \text { on } \Gamma_{1} \times(0, T), \\
\boldsymbol{\sigma} \cdot \nu=\mathbf{f}_{2} \quad \text { on } \Gamma_{2} \times(0, T), \\
-k_{i j} \frac{\partial \theta}{\partial x_{i}} n_{j}=k_{e}\left(\theta-\theta_{R}\right)+h_{\tau}\left(\left|\dot{\mathbf{u}}_{\tau}\right|\right), \quad \text { on } \Gamma_{3} \times(0, T), \\
\frac{\partial \xi}{\partial \nu}=0 \quad \text { on } \Gamma \times(0, T),
\end{gathered}
$$




$$
\begin{gathered}
\left\{\begin{array}{c}
\mathbf{u}_{\nu}=0, \quad\left|\boldsymbol{\sigma}_{\tau}\right| \leq g \\
\left|\boldsymbol{\sigma}_{\tau}\right|<g \Rightarrow \dot{\mathbf{u}}_{\tau}=0, \quad \text { on } \Gamma_{3} \times(0, T), \\
\left|\boldsymbol{\sigma}_{\tau}\right|=g \Rightarrow \exists \lambda \geq 0 \text { such that } \boldsymbol{\sigma}_{\tau}=-\lambda \dot{\mathbf{u}}_{\tau}
\end{array}\right. \\
\theta=0, \quad \text { on }\left(\Gamma_{1} \cup \Gamma_{2}\right) \times(0, T), \\
\mathbf{u}(0)=\mathbf{u}_{0}, \dot{\mathbf{u}}(0)=\mathbf{v}_{0}, \xi(0)=\xi_{0}, \theta(0)=\theta_{0}, \quad, \quad \text { in } \Omega,
\end{gathered}
$$

First, equations (2.1), (2.2) and (2.3) represent the elastic-viscoplastic constitutive law with damage and thermal effects, equation (2.4) represents the equation of motion where $\rho$ represents the mass density. Equations (2.5) and (2.6) represent the displacement and traction boundary condition, respectively. (2.7), (2.8) represent, respectively on $\Gamma$, a Fourier boundary condition for the temperature and an homogeneous Neumann boundary condition for the damage field on $\Gamma$. We assume that the contact is bilateral, therfore, the normal displacement $\mathbf{u}_{\nu}$ vanishes on $\Gamma_{3} \times(0, T)$. We involve the friction process with Tresca's friction law, where the friction yield limit is $g$, which is assumed to depend only on each point of $\Gamma_{3}, \dot{\mathbf{u}}_{\tau}$ denotes the tangential velocity and $\boldsymbol{\sigma}_{\tau}$ represent the tangential stress. The strong inequality holds in stick zone and the equality in slip zone. To simplify the notation, we do not indicate explcitely the dependence of various functions on the variable $x \in \Omega \cup \Gamma$ and $t \in[0, T]$. Equation (2.10) means that the temperature vanishes on $\left(\Gamma_{1} \cup \Gamma_{2}\right) \times(0, T)$. The functions $\mathbf{u}_{0}$, $\mathbf{v}_{0}, \xi_{0}$ and $\theta_{0}$ in $(2.11)$ are the initial data.

\section{Variational formulation and preliminaries}

In this section, we list the assumptions on the data and derive a variational formulation for the contact problem. To this end, we need to introduce some notations and preliminary material. For more details, we refer the reader to $[2,8]$. We denote by $\mathbb{S}^{d}$ the space of second order symmetric tensors on $\mathbb{R}^{d}(d=2,3)$, while $\|\cdot\|$ denotes the Euclidean norm.

Let $\Omega \subset \mathbb{R}^{d}$ be a bounded domain with Lipschitz boundary $\Gamma$ and let $\nu$ denote the unit outer normal on $\partial \Omega=\Gamma$. We shall use the notations

$$
\begin{array}{ll}
H=L^{2}(\Omega)^{d}=\left\{\mathbf{u}=\left(u_{i}\right): u_{i} \in L^{2}(\Omega)\right\}, & \mathcal{H}=\left\{\boldsymbol{\sigma}=\left(\sigma_{i j}\right): \sigma_{i j}=\sigma_{j i} \in L^{2}(\Omega)\right\}, \\
H^{1}(\Omega)^{d}=\left\{\mathbf{u}=\left(u_{i}\right) \in H: u_{i} \in H^{1}(\Omega)\right\}, & \mathcal{H}_{1}=\{\boldsymbol{\sigma} \in \mathcal{H}: \operatorname{div} \boldsymbol{\sigma} \in H\} .
\end{array}
$$

Here $\varepsilon: H^{1}(\Omega)^{d} \rightarrow \mathcal{H}$ and div $: \mathcal{H}_{1} \rightarrow H$ are the deformation and divergence operators, respectively, defined by

$$
\varepsilon(\mathbf{u})=\left(\varepsilon_{i j}(\mathbf{u})\right), \quad \varepsilon_{i j}(\mathbf{u})=\frac{1}{2}\left(u_{i, j}+u_{j, i}\right), \quad \operatorname{div} \boldsymbol{\sigma}=\left(\sigma_{i j, j}\right) .
$$

Here and below, the indices $i$ and $j$ run from 1 to $d$, the summation convention over repeated indices is used and the index that follows a comma indicates a partial derivative with respect to the corresponding component of the independent variable. The spaces $H, \mathcal{H}, H^{1}(\Omega)^{d}$ and $\mathcal{H}_{1}$ are real Hilbert spaces endowed with the canonical 
inner products given by:

where

$$
\begin{gathered}
(\mathbf{u}, \mathbf{v})_{H}=\int_{\Omega} u_{i} v_{i} d x, \quad \mathbf{u}, \mathbf{v} \in H, \quad(\boldsymbol{\sigma}, \boldsymbol{\tau})_{\mathcal{H}}=\int_{\Omega} \sigma_{i j} \tau_{i j} d x, \quad \forall \boldsymbol{\sigma}, \boldsymbol{\tau} \in \mathcal{H} \\
(\mathbf{u}, \mathbf{v})_{H^{1}(\Omega)^{d}}=\int_{\Omega} \mathbf{u . v} d x+\int_{\Omega} \nabla \mathbf{u} . \nabla \mathbf{v} d x \quad \forall \mathbf{u}, \mathbf{v} \in H^{1}(\Omega)^{d},
\end{gathered}
$$

$$
\begin{gathered}
\nabla \mathbf{v}=\left(v_{i, j}\right), \quad \forall \mathbf{v} \in H^{1}(\Omega)^{d} . \\
(\boldsymbol{\sigma}, \boldsymbol{\tau})_{\mathcal{H}_{1}}=(\boldsymbol{\sigma}, \boldsymbol{\tau})_{\mathcal{H}}+(\operatorname{div} \boldsymbol{\sigma}, \operatorname{div} \boldsymbol{\tau})_{H} \forall \boldsymbol{\sigma}, \boldsymbol{\tau} \in \mathcal{H}_{1},
\end{gathered}
$$

The associated norms are denoted by $\|\cdot\|_{H},\|\cdot\|_{\mathcal{H}},\|\cdot\|_{H^{1}}$ and $\|\cdot\|_{\mathcal{H}_{1}}$, respectively. Let $H_{\Gamma}=\left(H^{1 / 2}(\Gamma)\right)^{d}$ and $\left.\gamma: H^{1}(\Gamma)\right)^{d} \rightarrow H_{\Gamma}$ be the trace map. For every element $\mathbf{v} \in H^{1}(\Omega)^{d}$, we also use the notation $\mathbf{v}$ to denote the trace map $\gamma \mathbf{v}$ of $\mathbf{v}$ on $\Gamma$, and we denote by $v_{\nu}$ and $\mathbf{v}_{\tau}$ the normal and tangential components of $\mathbf{v}$ on $\Gamma$ given by

$$
v_{\nu}=\mathbf{v} \cdot \nu, \quad \mathbf{v}_{\tau}=\mathbf{v}-v_{\nu} \nu
$$

Similarly, for a regular (say $\mathcal{C}^{1}$ ) tensor field $\boldsymbol{\sigma}: \Omega \rightarrow \mathbb{S}^{d}$ we define its normal and tangential components by

$$
\boldsymbol{\sigma}_{\nu}=(\boldsymbol{\sigma} \nu) \cdot \boldsymbol{\nu}, \quad \boldsymbol{\sigma}_{\tau}=\boldsymbol{\sigma} \nu-\boldsymbol{\sigma}_{\nu} \boldsymbol{\nu}
$$

and for all $\boldsymbol{\sigma} \in \mathcal{H}_{1}$ the following Green's formula holds

$$
(\boldsymbol{\sigma}, \varepsilon(\mathbf{v}))_{\mathcal{H}}+(\operatorname{div} \boldsymbol{\sigma}, \mathbf{v})_{H}=\int_{\Gamma} \boldsymbol{\sigma} \nu \cdot \mathbf{v} d a \quad \forall \mathbf{v} \in H^{1}(\Omega)^{d} .
$$

Finally, for any real Hilbert space $X$, we use the classical notation for the spaces $L^{p}(0, T ; X)$ and $W^{k, p}(0, T ; X)$, where $1 \leqslant p \leqslant \infty$ and $k>1$. For $T>0$ we denote by $\mathcal{C}(0, T ; X)$ and $\mathcal{C}^{1}(0, T ; X)$ the space of continuous and continuously differentiable functions from $[0, T]$ to $X$, respectively, with the norms

$$
\begin{gathered}
\|\mathbf{f}\|_{\mathcal{C}(0, T ; X)}=\max _{t \in[0, T]}\|\mathbf{f}(t)\|_{X}, \\
\|\mathbf{f}\|_{\mathcal{C}^{1}(0, T ; X)}=\max _{t \in[0, T]}\|\mathbf{f}(t)\|_{X}+\max _{t \in[0, T]}\|\dot{\mathbf{f}}(t)\|_{X},
\end{gathered}
$$

respectively. Moreover, we use the dot above to indicate the derivative with respect to the time variable and if $X_{1}$ and $X_{2}$ are real Hilbert spaces then $X_{1} \times X_{2}$ denotes the product Hilbert space endowed with the canonical inner product $(\cdot, \cdot)_{X_{1} \times X_{2}}$.

Now, let $E$ denote the closed subspace of $H^{1}(\Omega)$ given by

$$
E=\left\{\gamma \in H^{1}(\Omega): \gamma=0 \quad \text { on } \quad \Gamma_{1} \cup \Gamma_{2}\right\}
$$

Let $V$ denote the closed subspace of $H_{1}$ defined by

$$
V=\left\{v \in H_{1}: v=0 \text { on } \Gamma_{1} \text { and } v_{\nu}=0 \text { on } \Gamma_{3}\right\}
$$

Since meas $\Gamma_{1}>0$, the following Korn's inequality holds:

$$
\|\varepsilon(\mathbf{v})\|_{\mathcal{H}} \geq c_{K}\|\mathbf{v}\|_{H_{1}} \quad \forall \mathbf{v} \in V
$$

where the constant $c_{K}$ denotes a positive constant which may depends only on $\Omega, \Gamma_{1}$ Over the space $V$ we consider the inner product given by

$$
(\mathbf{u}, \mathbf{v})_{V}=(\varepsilon(\mathbf{u}), \varepsilon(\mathbf{v}))_{\mathcal{H}}, \quad \forall \mathbf{u}, \mathbf{v} \in V .
$$


Let $\|\cdot\|_{V}$ be the associated norm. It follows from Korn's inequality (3.3) that the norms $\|\cdot\|_{H_{1}}$ and $\|\cdot\|_{V}$ are equivalent on $V$. Then $\left(V,\|\cdot\|_{V}\right)$ is a real Hilbert space. Moreover, by the Sobolev trace theorem and (3.3), there exists a constant $c_{0}>0$, depending only on $\Omega, \Gamma_{1}$ and $\Gamma_{3}$ such that

$$
\|\mathbf{v}\|_{L^{2}\left(\Gamma_{3}\right)^{d}} \leq c_{0}\|\mathbf{v}\|_{V} \quad \forall \mathbf{v} \in V .
$$

The mechanical problem may be formulated as follows.

In the study of the Problem $\mathbf{P}$, we consider the following assumptions:

The viscosity function $\mathcal{A}: \Omega \times \mathbb{S}^{d} \rightarrow \mathbb{S}^{d}$ satisfies:

(a) There exists $L_{\mathcal{A}}>0$ such that

$\left|\mathcal{A}\left(\boldsymbol{x}, \varepsilon_{1}\right)-\mathcal{A}\left(\boldsymbol{x}, \varepsilon_{2}\right)\right| \leq L_{\mathcal{A}}\left|\varepsilon_{1}-\varepsilon_{2}\right|$ for all $\varepsilon_{1}, \varepsilon_{2} \in \mathbb{S}^{d}$, a.e. $\boldsymbol{x} \in \Omega$.

(b) There exists $m_{\mathcal{A}}>0$ such that

$\left(\mathcal{A}\left(\boldsymbol{x}, \varepsilon_{1}\right)-\mathcal{A}\left(\boldsymbol{x}, \varepsilon_{2}\right)\right) \cdot\left(\varepsilon_{1}-\varepsilon_{2}\right) \geq m_{\mathcal{A}}\left|\varepsilon_{1}-\varepsilon_{2}\right|^{2}$ for all $\varepsilon_{1}, \varepsilon_{2} \in \mathbb{S}^{d}$, a.e. $\boldsymbol{x} \in \Omega$.

(c) The mapping $\boldsymbol{x} \mapsto \mathcal{A}(\boldsymbol{x}, \varepsilon)$ is Lebesgue measurable on $\Omega$, for any $\varepsilon \in \mathbb{S}^{d}$.

(d) The mapping $\boldsymbol{x} \mapsto \mathcal{A}(\boldsymbol{x}, \mathbf{0})$ is continuous on $\mathbb{S}^{d}$, a.e. $\boldsymbol{x} \in \Omega$.

The elasticity operator $\mathcal{G}: \Omega \times \mathbb{S}^{d} \times \mathbb{R} \rightarrow \mathbb{S}^{d}$ satisfies:

(a) There exists $L_{\mathcal{G}}>0$ such that $\left|\mathcal{G}\left(\boldsymbol{x}, \varepsilon_{1}, \boldsymbol{\xi}_{1}\right)-\mathcal{G}\left(\boldsymbol{x}, \varepsilon_{2}, \boldsymbol{\xi}_{2}\right)\right| \leq L_{\mathcal{G}}\left(\left|\varepsilon_{1}-\varepsilon_{2}\right|+\left|\boldsymbol{\xi}_{1}-\boldsymbol{\xi}_{2}\right|\right)$, for all $\varepsilon_{1}, \varepsilon_{2} \in \mathbb{S}^{d}$, for all $\boldsymbol{\xi}_{1}, \boldsymbol{\xi}_{2} \in \mathbb{R}$, a.e. $\boldsymbol{x} \in \Omega$.

(b) The mapping $\boldsymbol{x} \mapsto \mathcal{G}(\boldsymbol{x}, \varepsilon, \boldsymbol{\xi})$ is Lebesgue measurable on $\Omega$, for any $\varepsilon \in \mathbb{S}^{d}$, and for all $\boldsymbol{\xi} \in \mathbb{R}$.

(c) The mapping $\boldsymbol{x} \mapsto \mathcal{G}(\boldsymbol{x}, \mathbf{0}, \mathbf{0})$ belongs to $\mathcal{H}$.

The visco-plasticity operator $\mathcal{F}: \Omega \times \mathbb{S}^{d} \times \mathbb{S}^{d} \rightarrow \mathbb{S}^{d}$ satisfies:

(a) There exists $L_{\mathcal{F}}>0$ such that

$\left|\mathcal{F}\left(\boldsymbol{x}, \boldsymbol{\sigma}_{1}, \varepsilon_{1}\right)-\mathcal{F}\left(\boldsymbol{x}, \boldsymbol{\sigma}_{2}, \varepsilon_{2}\right)\right| \leq L_{\mathcal{F}}\left(\left|\boldsymbol{\sigma}_{1}-\boldsymbol{\sigma}_{2}\right|+\left|\varepsilon_{1}-\varepsilon_{2}\right|\right)$,

for all $\boldsymbol{\sigma}_{1}, \boldsymbol{\sigma}_{2}, \varepsilon_{1}, \varepsilon_{2} \in \mathbb{S}^{d}$ a.e. $\boldsymbol{x} \in \Omega$,

(b) The mapping $\boldsymbol{x} \mapsto \mathcal{F}(\boldsymbol{x}, \boldsymbol{\sigma}, \varepsilon)$ is Lebesgue measurable on $\Omega$, for any $\boldsymbol{\sigma}, \varepsilon \in \mathbb{S}^{d}$

(c) The mapping $\boldsymbol{x} \mapsto \mathcal{F}(\boldsymbol{x}, \mathbf{0}, \mathbf{0})$ belongs to $\mathcal{H}$.

The damage source function $S: \Omega \times \mathbb{S}^{d} \times \mathbb{R} \rightarrow \mathbb{S}^{d}$ satisfies:

(a) There exists $L_{S}>0$ such that $\left|S\left(\boldsymbol{x}, \boldsymbol{\varepsilon}_{1}, \boldsymbol{\xi}_{1}\right)-S\left(\boldsymbol{x}, \boldsymbol{\varepsilon}_{2}, \boldsymbol{\xi}_{2}\right)\right| \leq L_{S}\left(\left|\varepsilon_{1}-\varepsilon_{2}\right|+\left|\boldsymbol{\zeta}_{1}-\boldsymbol{\zeta}_{2}\right|\right)$,

for all $\varepsilon_{1}, \varepsilon_{2} \in \mathbb{S}^{d}, \boldsymbol{\xi}_{1}, \boldsymbol{\xi}_{2} \in \mathbb{R}$, a.e. $\boldsymbol{x} \in \Omega$.

(b) The mapping $\boldsymbol{x} \mapsto S(\boldsymbol{x}, \boldsymbol{\varepsilon}, \boldsymbol{\xi})$ is Lebesgue measurable on $\Omega$,

for any $\varepsilon \in \mathbb{S}^{d}$, and for all $\boldsymbol{\xi} \in \mathbb{R}$.

(c) The mapping $\boldsymbol{x} \mapsto S(\boldsymbol{x}, \mathbf{0}, \mathbf{0})$ belongs to $\mathcal{H}$. 
The thermal expansion operator $C_{e}: \Omega \times \mathbb{R} \rightarrow \mathbb{R}$ satisfies:

(a) There exists $L_{C_{e}}>0$ such that $\left|C_{e}\left(\boldsymbol{x}, \boldsymbol{\theta}_{1}\right)-C_{e}\left(\boldsymbol{x}, \boldsymbol{\theta}_{2}\right)\right| \leq L_{C_{e}}\left|\boldsymbol{\theta}_{1}-\boldsymbol{\theta}_{2}\right|$ for all $\boldsymbol{\theta}_{1}, \boldsymbol{\theta}_{2} \in \mathbb{R}$, a.e. $\boldsymbol{x} \in \Omega$.

(b) $C_{e}=\left(c_{i j}\right), c_{i j}=c_{j i} \in L^{\infty}(\Omega)$.

(c) The mapping $\boldsymbol{x} \mapsto C_{e}(\boldsymbol{x}, \boldsymbol{\theta})$ is Lebesgue measurable on $\Omega$, for any $\boldsymbol{\theta} \in \mathbb{R}$.

(d) The mapping $\boldsymbol{x} \mapsto C_{e}(\boldsymbol{x}, \mathbf{0}) \in \mathcal{H}$.

The thermal conductivity operator $K: \Omega \times \mathbb{R} \rightarrow \mathbb{R}$ satisfies:

(a) There exists $L_{K}>0$ such that $\left|K\left(\boldsymbol{x}, r_{1}\right)-K\left(\boldsymbol{x}, r_{2}\right)\right| \leq L_{K}\left|r_{1}-r_{2}\right|$, for all $r_{1}, r_{2} \in \mathbb{R}$, a.e. $\boldsymbol{x} \in \Omega$.

(b) $k_{i j}=k_{j i} \in L^{\infty}(\Omega), k_{i j} \alpha_{i} \alpha_{j} \leq c_{k} \alpha_{i} \alpha_{j}$ for some $c_{k}>0$, for all $\left(\alpha_{i}\right) \in \mathbb{R}$.

(c) The mapping $\boldsymbol{x} \mapsto k(\boldsymbol{x}, \mathbf{0})$ belongs to $L^{2}(\Omega)$.

We assume that the tangential function $h_{\tau}: \Gamma_{3} \times \mathbb{R} \rightarrow \mathbb{R}_{+}$satisfies:

(a) There exists $L_{\tau}>0$ such that $\left|h_{\tau}\left(\boldsymbol{x}, r_{1}\right)-h_{\tau}\left(\boldsymbol{x}, r_{2}\right)\right| \leq L_{\tau}\left|r_{1}-r_{2}\right|$ for all $r_{1}, r_{2} \in \mathbb{R}_{+}$, a.e. $\boldsymbol{x} \in \Omega$.

(b) The mapping $\boldsymbol{x} \mapsto h_{\tau}(\boldsymbol{x}, r)$ is Lebesgue measurable on $\Gamma_{3}$ for all $r \in \mathbb{R}_{+}$.

(c) The mapping $\boldsymbol{x} \mapsto h_{\tau}(\boldsymbol{x}, 0)$ belongs to $L^{2}\left(\Gamma_{3}\right)$.

A concrete example of a tangential function $h_{\tau}$ is given by

$$
h_{\tau}(x, r)=\lambda(x) r, \forall r \in \mathbb{R}_{+} \text {, a.e } x \in \Gamma_{3},
$$

where $\lambda \in L^{\infty}\left(\Gamma_{3}, \mathbb{R}_{+}\right)$represents some rate coefficient for the gradient of the temperature.

The masse density satisfies

$$
\rho \in L^{\infty}(\Omega) \text {, there exists } \rho^{*}>0 \text { such that } \rho(x) \geq \rho^{*} \text {, a.e } x \in \Omega
$$

and

$$
g \in L^{\infty}\left(\Gamma_{3}\right), \quad g \geq 0, \text { a.e. on } \Gamma_{3}
$$

We also suppose the following regularities

$$
\mathbf{f}_{0} \in L^{2}(0, T ; H), \quad \mathbf{f}_{2} \in L^{2}\left(0, T ; L^{2}\left(\Gamma_{2}\right)^{d}\right), \mathbf{q} \in L^{2}\left(0, T ; L^{2}(\Omega)\right) .
$$

The boundary and initial data satisfy

$$
\begin{gathered}
\mathbf{u}_{0} \in V, \mathbf{v}_{0} \in H \\
\xi_{0} \in F \\
\theta_{0} \in E \\
\theta_{R} \in L^{2}\left(0, T ; L^{2}\left(\Gamma_{3}\right)\right) \\
k_{e} \in L^{\infty}\left(\Omega, \mathbb{R}_{+}\right)
\end{gathered}
$$


The function $r: V \rightarrow L^{2}(\Omega)$ satisfies that there exists a constant $L_{r}>0$ such that

$$
\begin{aligned}
\left|r\left(\mathbf{v}_{1}, \xi_{1}\right)-r\left(\mathbf{v}_{2}, \xi_{2}\right)\right|_{L^{2}(\Omega)} & \left.\leq L_{r}\left(\left|\mathbf{v}_{1}-\mathbf{v}_{2}\right|_{V}+\mid \xi_{1}-\xi_{2}\right) \mid\right) \\
\forall \mathbf{v}_{1}, \mathbf{v}_{2} & \in V, \quad \xi_{1}, \xi_{2} \in \mathbb{R}
\end{aligned}
$$

We use a modified inner product on $H=L^{2}(\Omega)^{d}$ given by

$$
((\mathbf{u}, \mathbf{v}))_{H}=(\rho \mathbf{u}, \mathbf{v})_{H}, \quad \forall \mathbf{u}, \mathbf{v} \in H
$$

that is, it is weighted with $\rho$. We let $\|\cdot\|_{H}$ be the associated norm, i.e

$$
\|\mathbf{v}\|_{H}=(\rho \mathbf{v}, \mathbf{v})_{H}^{\frac{1}{2}}, \quad \forall \mathbf{v} \in H
$$

The notation $(\cdot, \cdot)_{V \prime \times V}$ represent the duality pairing between $V^{\prime}$ and $V$. Then, we have

$$
(\mathbf{u}, \mathbf{v})_{V^{\prime} \times V}=((\mathbf{u}, \mathbf{v}))_{H}, \quad \forall \mathbf{u} \in H, \quad \forall \mathbf{v} \in V
$$

It follows from assumption (3.13) that $\|\cdot\|_{H}$ and $|\cdot|_{H}$ are equivalent norms on $H$, and also the inclusion mapping of $\left(V,|\cdot|_{V}\right)$ into $\left(H,\|\cdot\|_{H}\right)$ is continuous and dense. We denote by $V^{\prime}$ the dual space of $V$. Identifying $H$ with its own dual, we can write the Gelfand triple

$$
V \subset H \subset V^{\prime}
$$

From assumption (3.15) we define $\mathbf{f}(t) \in V$ for a.e. $t \in(0, T)$ by

$$
(\mathbf{f}(t), \mathbf{v})_{V \prime \times V}=\int_{\Omega} \mathbf{f}_{0}(t) \cdot \mathbf{v} d x+\int_{\Gamma_{2}} \mathbf{f}_{2}(t) \cdot \mathbf{v} d a \quad \forall \mathbf{v} \in \boldsymbol{V}
$$

and note that

$$
\mathbf{f} \in L^{2}(0, T ; V) .
$$

We define the bilinear form $j: H^{1}(\Omega) \times H^{1}(\Omega) \rightarrow \mathbb{R}$

$$
a(\varsigma, \zeta)=\kappa \int_{\Omega} \nabla \varsigma \cdot \nabla \zeta d x
$$

Next we define the functional $j: V \rightarrow \mathbb{R}$ by

$$
j(\mathbf{v})=\int_{\Gamma_{3}} g\left|\mathbf{v}_{\tau}\right| d a, \quad \forall \mathbf{v} \in \boldsymbol{V} .
$$

By using a standard arguments, we obtain the following variational formulation of the mechanical problem (2.1)-(2.11). 
Problem PV. Find a displacement field $\mathbf{u}:[0, T] \rightarrow \boldsymbol{V}$, a stress field $\boldsymbol{\sigma}:[0, T] \rightarrow \mathcal{H}$, a temperature $\theta:[0, T] \rightarrow E$, a damage $\xi:[0, T] \rightarrow E_{1}$, such that for a.e. $t \in(0, T)$

$$
\begin{gathered}
\boldsymbol{\sigma}(t)=\mathcal{A} \varepsilon(\dot{\mathbf{u}}(t))+\mathcal{G}(\varepsilon(\mathbf{u}(t), \xi(t))) \\
+\int_{0}^{t} \mathcal{F}(\boldsymbol{\sigma}(s)-\mathcal{A} \varepsilon(\dot{\mathbf{u}}(s)), \boldsymbol{\varepsilon}(\mathbf{u}(s))) d s-C_{e} \theta(t) \\
(\ddot{\mathbf{u}}(t), w-\dot{\mathbf{u}}(t))_{V^{\prime} \times V}+(\boldsymbol{\sigma}(t), \varepsilon(w-\dot{\mathbf{u}}(\mathbf{t})))_{\mathcal{H}} \\
+j(w)-j(\dot{\mathbf{u}}(t)) \geq(\mathbf{f}(t), w-\dot{\mathbf{u}}(t))_{V^{\prime} \times V}, \quad \forall w \in V \\
\dot{\theta}(t)+K \theta(t)=R \dot{\mathbf{u}}(t)+Q(t), \quad \text { in } E^{\prime} \\
(\dot{\xi}(t), \zeta-\xi(t))_{L^{2}(\Omega)}+a(\xi(t), \zeta-\xi(t)) \\
\geq(S(\boldsymbol{\varepsilon}(\mathbf{u}(t)), \xi(t)), \zeta-\xi(t))_{L^{2}(\Omega)} \\
\text { for all } \xi(t) \in F, \zeta \in F \text { and } t \in(0, T) \\
\mathbf{u}(0)=\mathbf{u}_{0}, \dot{\mathbf{u}}(0)=\mathbf{v}_{0}, \theta(0)=\theta_{0}, \xi(0)=\xi_{0},
\end{gathered}
$$

where $Q:[0, T] \rightarrow E^{\prime}, K: E \rightarrow E^{\prime}$, and $\mathcal{R}: V \rightarrow E^{\prime}$ are given by

$$
\begin{gathered}
(Q(t), \eta)_{E \prime \times E}=\int_{\Gamma_{3}} k_{e} \theta_{R}(t) \eta d a+\int_{\Omega} q(t) \eta d x \\
(K \tau, \eta)_{E \prime \times E}=\sum_{i, j=1}^{d} \int_{\Omega} k_{i j} \frac{\partial \tau}{\partial x_{j}} \frac{\partial \eta}{\partial x_{i}} d x+\int_{\Gamma_{3}} k_{e} \tau \eta d a \\
(R v, \eta)_{E \prime \times E}=\int_{\Omega} r(v) \eta d x+\int_{\Gamma_{3}} h_{r}\left(\left|v_{r}\right|\right) \eta d a
\end{gathered}
$$

for all $v \in V, \eta, \tau \in E$.

We notice that the variational Problem $\mathbf{P V}$ is formulated in terms of a displacement field, a stress field, a temperature, and damage. The existence of the unique solution of problem $\mathbf{P V}$ is stated and proved in the next section.

\section{Existence and uniqueness result}

The main results are stated by the following theorems.

Theorem 4.1. Assume that (3.6)-(3.21) hold and, then there exists a unique solution $\{\mathbf{u}, \boldsymbol{\sigma}, \theta, \xi\}$ to problem $P V$. Moreover, the solution has the regularity

$$
\begin{gathered}
\mathbf{u} \in W^{1,2}(0, T ; V) \cap \mathcal{C}^{1}(0, T ; H) \cap W^{2,2}\left(0, T ; V^{\prime}\right), \\
\boldsymbol{\sigma} \in L^{2}(0, T ; \mathcal{H}), \operatorname{div} \boldsymbol{\sigma} \in L^{2}\left(0, T ; V^{\prime}\right), \\
\theta \in \mathcal{C}\left(0, T ; L^{2}(\Omega)\right) \cap L^{2}(0, T ; E) \cap W^{1,2}\left(0, T ; E^{\prime}\right), \\
\xi \in W^{1,2}\left(0, T ; L^{2}(\Omega)\right) \cap L^{2}\left(0, T ; H^{1}(\Omega)\right) .
\end{gathered}
$$

We conclude that under the assumptions, the mechanical problem has a unique weak solution with the regularity. 
The proof of this theorem will be carried out in several steps. It is based on arguments of first order evolution nonlinear inequalities, evolution equations, and fixed point arguments.

Let $\boldsymbol{\eta} \in L^{2}\left(0, T ; V^{\prime}\right)$ be given, in the first step, we consider the following variational problem.

Problem $\mathbf{P V} \mathbf{1}_{\eta}$. Find a displacement field $\mathbf{u}_{\eta}:[0, T] \rightarrow \boldsymbol{V}$, such that

$$
\begin{gathered}
\left(\ddot{\mathbf{u}}_{\eta}(t), w-\dot{\mathbf{u}}_{\eta}(t)\right)_{V^{\prime} \times V}+\left(\mathcal{A} \varepsilon\left(\dot{\mathbf{u}}_{\eta}(\mathbf{t})\right), \varepsilon\left(w-\dot{\mathbf{u}}_{\eta}(\mathbf{t})\right)\right)_{\mathcal{H}}+ \\
(\boldsymbol{\sigma}(t), \varepsilon(w-\dot{\mathbf{u}}(\mathbf{t})))_{\mathcal{H}}+j(w)-j\left(\dot{\mathbf{u}}_{\eta}(t)\right)+\left(\eta(t), w-\dot{\mathbf{u}}_{\eta}(t)\right)_{V^{\prime} \times V} \\
\geq\left(\mathbf{f}(t), w-\dot{\mathbf{u}}_{\eta}(t)\right)_{V^{\prime} \times V}, \quad \forall w \in V \\
\mathbf{u}_{\eta}(0)=\mathbf{u}_{0}, \dot{\mathbf{u}}_{\eta}(0)=\mathbf{v}_{0}
\end{gathered}
$$

We define $\mathbf{f}_{\eta}(t) \in V^{\prime}$ for a.e.t $\in[0, T]$ by

$$
\left(\mathbf{f}_{\eta}(t), w\right)_{V^{\prime} \times V}=(\mathbf{f}(t)-\eta(t), w)_{V^{\prime} \times V} .
$$

we deduce that

$$
\mathbf{f}_{\eta} \in L^{2}\left(0, T ; V^{\prime}\right) .
$$

We define the operator $\mathbf{A}: V \rightarrow V^{\prime}$ by

$$
(\mathbf{A v}, w)_{V^{\prime} \times V}=(\mathcal{A} \varepsilon(\mathbf{v}), \varepsilon(w))_{\mathcal{H}}, \quad \forall \mathbf{v}, w \in V .
$$

We consider the following variational inequality.

Problem $\mathbf{Q} \mathbf{V}_{\eta}$. Find a displacement field $\mathbf{v}_{\eta}:[0, T] \rightarrow \boldsymbol{V}$, such that

$$
\begin{gathered}
\left(\dot{\mathbf{v}}_{\eta}(t), w-\mathbf{v}_{\eta}(t)\right)_{V^{\prime} \times V}+\left(\mathcal{A} \mathbf{v}_{\eta}(\mathbf{t}), w-\mathbf{v}_{\eta}(\mathbf{t})\right)_{V^{\prime} \times V}+j(w)-j\left(\mathbf{v}_{\eta}(t)\right) \\
\geq\left(\mathbf{f}_{\eta}(t), w-\mathbf{v}_{\eta}(t)\right)_{V^{\prime} \times V} \quad \forall w \in V, \text { a.e.t } \in[0, T], \\
\mathbf{v}_{\eta}(0)=\mathbf{v}_{0} .
\end{gathered}
$$

In the study of Problem $\mathbf{Q} \mathbf{V}_{\eta}$, we have the following result.

Lemma 4.2. For all $\eta \in L^{2}\left(0, T ; V^{\prime}\right), \mathbf{Q V}_{\eta}$ has a unique solution with the regularity

$$
\mathbf{v}_{\eta} \in \mathcal{C}(0, T ; H) \cap L^{2}(0, T ; V) \cap W^{1,2}\left(0, T ; V^{\prime}\right),
$$

Proof. We begin by the step of regularization (see[8]). We define

$$
h(t)=\mathbf{f}_{\eta}(t), \quad t \in[0, T]
$$

and for all $\varepsilon>0$

$$
j_{\varepsilon}(w)=\int_{\Gamma_{3}} g \sqrt{\left|w_{r}\right|^{2}+\varepsilon^{2}} d a, \quad \forall w \in V .
$$

After some algebra, for all $\varepsilon>0, j_{\varepsilon}$ is convex and $\mathcal{C}^{1}$ on $V$, and its Fréchet derivative satisfies

$$
\exists C>0, \quad \forall w \in V,\left|j_{\varepsilon}^{\prime}(w)\right|_{V} \leq C|g|_{L^{2}\left(\Gamma_{3}\right)} .
$$

From (3.6) and the monotonicity of $j_{\varepsilon}^{\prime}$, it follows from classical first order evolution equation that

such that

$$
\forall \varepsilon>0, \mathbf{v}_{\eta}^{\varepsilon} \in L^{2}(0, T ; V) \cap W^{1,2}\left(0, T ; V^{\prime}\right)
$$

$$
\left\{\begin{array}{l}
\dot{\mathbf{v}}_{\eta}^{\varepsilon}(t)+\left(\mathcal{A} \mathbf{v}_{\eta}^{\varepsilon}(t)+j_{\varepsilon}^{\prime}\left(\mathbf{v}_{\eta}^{\varepsilon}(t)\right)\right)=h(t) \text { in } V^{\prime}, \quad \text { a.e.t } \in[0, T] \\
\mathbf{v}_{\eta}^{\varepsilon}(0)=0
\end{array}\right.
$$


Then, we obtain

$$
\begin{aligned}
& \left(\dot{\mathbf{v}}_{\eta}^{\varepsilon}(t), w-\mathbf{v}_{\eta}^{\varepsilon}(t)\right)_{V^{\prime} \times V}+\left(\mathcal{A} \mathbf{v}_{\eta}^{\varepsilon}(t), w-\mathbf{v}_{\eta}^{\varepsilon}(t)\right)_{V^{\prime} \times V}+j_{\varepsilon}(w)-j_{\varepsilon}\left(\mathbf{v}_{\eta}^{\varepsilon}(t)\right) \\
& \geq\left(h(t), w-\mathbf{v}_{\eta}^{\varepsilon}(t)\right)_{V^{\prime} \times V}, \quad \forall w \in V, \text { a.e.t } \in[0, T]
\end{aligned}
$$

From (4.12), we have

$$
\begin{aligned}
& \left(\dot{\mathbf{v}}_{\eta}^{\varepsilon}(t), \mathbf{v}_{\eta}^{\varepsilon}(t)\right)_{V^{\prime} \times V}+\left(\mathcal{A} \mathbf{v}_{\eta}^{\varepsilon}(t), \mathbf{v}_{\eta}^{\varepsilon}(t)\right)_{V^{\prime} \times V}+\left(j_{\varepsilon}\left(\mathbf{v}_{\eta}^{\varepsilon}(t)\right), \mathbf{v}_{\eta}^{\varepsilon}(t)\right)_{V^{\prime} \times V} \\
& =\left(h(t), \mathbf{v}_{\eta}^{\varepsilon}(t)\right)_{V^{\prime} \times V}, \quad \text { a.e.t } \in[0, T]
\end{aligned}
$$

Using (3.6), and the monotony of $j_{\varepsilon}^{\prime}$, we deduce that

$$
\exists C>0, \forall t \in[0, T], \quad\left|\mathbf{v}_{\eta}^{\varepsilon}(t)\right|_{H} \leq C, \int_{0}^{T}\left|\mathbf{v}_{\eta}^{\varepsilon}(t)\right|_{V}^{2} d t \leq C, \int_{0}^{T}\left|\dot{\mathbf{v}}_{\eta}^{\varepsilon}(t)\right|_{V}^{2} d t \leq C .
$$

Using a subsequence to find that

$$
\left\{\begin{array}{l}
\mathbf{v}_{\eta}^{\varepsilon} \rightarrow \mathbf{v}_{\eta} \text { weakly in } L^{2}(0, T ; V) \text { and star weakly in } L^{2}(0, T ; H), \\
\dot{\mathbf{v}}_{\eta}^{\varepsilon} \rightarrow \dot{\mathbf{v}}_{\eta} \text { star weakly in } L^{2}(0, T ; V \prime) .
\end{array}\right.
$$

It follows that

$$
\mathbf{v}_{\eta} \in \mathcal{C}(0, T ; H) \text { and } \mathbf{v}_{\eta}^{\varepsilon}(t) \rightarrow \mathbf{v}_{\eta}(t) \text { weakly in } H, \forall t \in[0, T]
$$

Integrating (4.13), we have $\forall w \in L^{2}(0, T ; V)$

$$
\begin{aligned}
& \int_{0}^{T}\left(\dot{\mathbf{v}}_{\eta}^{\varepsilon}, w\right)_{V^{\prime} \times V} d t+\int_{0}^{T}\left(\mathcal{A} \mathbf{v}_{\eta}^{\varepsilon}, w\right)_{V^{\prime} \times V} d t+\int_{0}^{T} j_{\varepsilon}(w) d t \\
& \geq \int_{0}^{T}\left(\dot{\mathbf{v}}_{\eta}^{\varepsilon}, \mathbf{v}_{\eta}^{\varepsilon}\right)_{V^{\prime} \times V} d t+\int_{0}^{T}\left(\mathcal{A} \mathbf{v}_{\eta}^{\varepsilon}, \mathbf{v}_{\eta}^{\varepsilon}\right)_{V^{\prime} \times V} d t \\
& +\int_{0}^{T} j_{\varepsilon}\left(\mathbf{v}_{\eta}^{\varepsilon}\right) d t+\int_{0}^{T}\left(h, w-\mathbf{v}_{\eta}^{\varepsilon}\right)_{V^{\prime} \times V} d t \\
& \geq \frac{1}{2}\left|\mathbf{v}_{\eta}^{\varepsilon}(T)\right|_{H}^{2}-\frac{1}{2}\left|\mathbf{v}_{\eta}^{\varepsilon}(0)\right|_{H}^{2}+\int_{0}^{T}\left(\mathcal{A} \mathbf{v}_{\eta}^{\varepsilon}, \mathbf{v}_{\eta}^{\varepsilon}\right)_{V^{\prime} \times V} d t \\
& +\int_{0}^{T} j_{\varepsilon}\left(\mathbf{v}_{\eta}^{\varepsilon}\right) d t+\int_{0}^{T}\left(h, w-\mathbf{v}_{\eta}^{\varepsilon}\right)_{V^{\prime} \times V} d t
\end{aligned}
$$

From (4.14), (4.15) and the weak lower semicontinuity, we obtain that for all $w \in$ $L^{2}(0, T ; V)$ :

$$
\begin{aligned}
& \int_{0}^{T}\left(\dot{\mathbf{v}}_{\eta}, w-\mathbf{v}_{\eta}\right)_{V^{\prime} \times V} d t+\int_{0}^{T}\left(\mathcal{A} \mathbf{v}_{\eta}, w-\mathbf{v}_{\eta}\right)_{V^{\prime} \times V} d t+\int_{0}^{T} j(w)-j\left(\mathbf{v}_{\eta}\right) d t \\
& \geq \int_{0}^{T}\left(h, w-\mathbf{v}_{\eta}\right)_{V^{\prime} \times V} .
\end{aligned}
$$

The previous inequality implies (see [8]) that

$$
\begin{aligned}
& \left(\dot{\mathbf{v}}_{\eta}(t), w-\mathbf{v}_{\eta}(t)\right)_{V^{\prime} \times V}+\left(\mathcal{A} \mathbf{v}_{\eta}(t), w-\mathbf{v}_{\eta}(t)\right)_{V^{\prime} \times V}+j(w)-j\left(\mathbf{v}_{\eta}(t)\right) \\
& \geq\left(h(t), w-\mathbf{v}_{\eta}(t)\right)_{V^{\prime} \times V}, \quad \forall w \in V, \text { a.e.t } \in[0, T] .
\end{aligned}
$$

We conclude that Problem $\mathbf{Q} \mathbf{V}_{\eta}$ has at least a solution $\mathbf{v}_{\eta} \in \mathcal{C}(0, T ; H) \cap L^{2}(0, T ; V) \cap$ $W^{1,2}\left(0, T ; V^{\prime}\right)$. For the uniqueness, let $\mathbf{v}_{\eta}^{1}, \mathbf{v}_{\eta}^{2}$ be two solutions of $\mathbf{Q} \mathbf{V}_{\eta}$. We use (4.10) to obtain for a.e.t $\in[0, T]$,

$$
\left(\dot{\mathbf{v}}_{\eta}^{2}(t)-\dot{\mathbf{v}}_{\eta}^{1}(t), \mathbf{v}_{\eta}^{2}(t)-\mathbf{v}_{\eta}^{1}(t)\right)_{V^{\prime} \times V}+\left(\mathcal{A} \mathbf{v}_{\eta}^{2}(t)-\mathcal{A} \mathbf{v}_{\eta}^{1}(t), \mathbf{v}_{\eta}^{2}(t)-\mathbf{v}_{\eta}^{1}(t)\right)_{V^{\prime} \times V} \leq 0
$$


Integrating the previous inequality, using (3.6)and (4.9), we find

$$
\frac{1}{2}\left|\mathbf{v}_{\eta}^{2}(t)-\mathbf{v}_{\eta}^{1}(t)\right|_{H}^{2}+m_{\mathcal{A}} \int_{0}^{t}\left|\mathbf{v}_{\eta}^{2}(s)-\mathbf{v}_{\eta}^{1}(s)\right|_{V}^{2} d s \leq 0
$$

which implies

$$
\mathbf{v}_{\eta}^{1}=\mathbf{v}_{\eta}^{2}
$$

Let now $\mathbf{u}_{\eta}:[0, T] \rightarrow V$ be the function defined by

$$
\mathbf{u}_{\eta}(t)=\int_{0}^{t} \mathbf{v}_{\eta}(s) d s+\mathbf{u}_{0}, \quad \forall t \in[0, T] .
$$

In the study of Problem $\mathbf{P V} \mathbf{1}_{\eta}$, we have the following result.

Lemma 4.3. $\mathbf{P V 1} \mathbf{1}_{\eta}$ has a unique solution satisfying the regularity expressed in (4.1)

Proof. The proof of Lemma 4.3 is a consequence of Lemma 4.2 and the relation (4.17).

In the second step, we use the displacement field $\mathbf{u}_{\eta}$ obtained in Lemma 4.3 to consider the following variational problem.

Problem PV2 $\mathbf{2}_{\eta}$. Find a temperature field $\theta_{\eta}:[0, T] \rightarrow \boldsymbol{E}$, such that

$$
\begin{aligned}
\dot{\theta}_{\eta}(t)+K \theta_{\eta}(t) & =R \dot{\mathbf{u}}_{\eta}(t)+Q(t), \quad \text { in } E^{\prime}, \quad \text { a.e.t } \in[0, T] \\
\theta_{\eta}(0) & =\theta_{0}
\end{aligned}
$$

In the study of Problem $\mathbf{P V 2} \boldsymbol{2}_{\eta}$, we have the following result.

Lemma 4.4. $P V 2_{\eta}$ has a unique solution satisfying

$$
\theta_{\eta} \in C\left(0, T ; L^{2}(\Omega)\right) \cap L^{2}(0, T ; E) \cap W^{1,2}\left(0, T ; E^{\prime}\right) .
$$

Moreover, $\exists C>0$ such that $\forall \eta_{1}, \eta_{2} \in L^{2}\left(0, T ; V^{\prime}\right)$

$$
\left|\theta_{\eta_{1}}(t)-\theta_{\eta_{2}}(t)\right|_{L^{2}(\Omega)}^{2} \leq C \int_{0}^{t}\left|\eta_{1}(s)-\eta_{2}(s)\right|_{V}^{2} d s, \quad \forall t \in[0, T] .
$$

Proof. The result follows from classical first order evolution equation given in [2]. Here the Gelfand triple is given by

$$
E \subset L^{2}(\Omega)=\left(L^{2}(\Omega)\right)^{\prime} \subset E^{\prime} .
$$

The operator $K$ is linear and coercive. By Korn's inequality, we have

$$
(K \tau, \tau)_{E^{\prime} \times E} \geq C|\tau|_{E}^{2} .
$$

Here and below, $C>0$ denotes a generic constant whose value may change from line to line. 
Let $\eta \in \mathcal{C}\left(0, T ; L^{2}(\Omega)\right)$ be given and consider the following variational problem for the damage filed.

Problem $\mathbf{P V} \mathbf{3}_{\eta}$. Find the damage field $\xi_{\eta}:[0, T] \rightarrow H^{1}(\Omega)$ such that $\xi_{\eta}(t) \in F$ and

$$
\begin{gathered}
\left(\dot{\xi}_{\eta}(t), \zeta-\xi_{\eta}(t)\right)_{L^{2}(\Omega)}+a\left(\xi_{\eta}(t), \zeta-\xi_{\eta}(t)\right) \\
\geq\left(S\left(\boldsymbol{\varepsilon}(\mathbf{u}(t)), \xi_{\eta}(t)\right), \zeta-\xi_{\eta}(t)\right)_{L^{2}(\Omega)} \\
\xi_{\eta}(0)=\xi_{0}
\end{gathered}
$$

for all $\xi(t) \in F, \zeta \in F$ and $t \in(0, T)$

Note that if $f \in H$ then

$$
(f, v)_{V^{\prime} \times V}=(f, v)_{H}, \forall v \in H .
$$

Theorem 4.5. Let $V \subset H \subset V^{\prime}$ be a Gelfand triple. Let $K$ be a nonempty, closed, and convex set of $V$. Assume that $a(\cdot, \cdot): V \times V \rightarrow \mathbb{R}$ is a continuous and symmetric bilinear form such that for some constants $\zeta>0$ and $c_{0}$,

$$
a(v, v)=c_{0}\|v\|_{H}^{2} \geqslant \zeta\|v\|_{V}^{2}, \forall v \in H .
$$

Then, for every $u_{0} \in K$ and $f \in L^{2}(0, T ; H)$, there exists a unique function $u \in$ $H^{1}(0, T ; H) \cap L^{2}(0, T ; V)$ such that $u(0)=u_{0}, u(t) \in K$ for all $t \in[0, T]$, and for almost all $t \in(0, T)$,

$$
(\dot{u}(t), v-u(t))_{V^{\prime} \times V}+a(u(t), v-u(t)) \geqslant(f(t), v-u(t))_{H}, \forall v \in K,
$$

We apply this theorem to Problem $\mathbf{P V} \mathbf{3}_{\eta}$.

Lemma 4.6. There exists a unique solution $\xi_{\eta}$ to the auxiliary problem $\mathbf{P V} \mathbf{3}_{\eta}$ such that:

$$
\xi_{\eta} \in W^{1,2}\left(0, T ; L^{2}(\Omega)\right) \cap L^{2}\left(0, T ; H^{1}(\Omega)\right) .
$$

The above lemma follows from a standard result for parabolic variational inequalities.

Proof. The inclusion mapping of $\left(H^{1}(\Omega),\|\cdot\|_{H^{1}(\Omega)}\right)$ into $\left(L^{2}(\Omega),\|\cdot\|_{L^{2}(\Omega)}\right.$ is continuous and its range is dense. We denote by $\left(H^{1}(\Omega)\right)^{\prime}$ the dual space of $H^{1}(\Omega)$ and, identifying the dual of $L^{2}(\Omega)$ with itself, we can write the Gelfand triple

$$
H^{1}(\Omega) \subset L^{2}(\Omega) \subset\left(H^{1}(\Omega)\right)^{\prime} .
$$

We use the notation $(\cdot, \cdot)_{\left(H^{1}(\Omega)\right)^{\prime} \times H^{1}(\Omega)}$ to represent the duality pairing between $\left(H^{1}(\Omega)\right)^{\prime}$ and $H^{1}(\Omega)$. we have

$$
(\xi, \beta)_{\left(H^{1}(\Omega)\right)^{\prime} \times H^{1}(\Omega)}=(\xi, \beta)_{L^{2}(\Omega)}, \forall \xi \in L^{2}(\Omega), \beta \in H^{1}(\Omega)
$$

and we note that $F$ is a closed convex set in $H^{1}(\Omega)$. Then, using the definition $(3.23)$ of the bilinear form $a$, and the fact that $\xi_{\eta} \in F$.

In the fourth step, we use the displacement field $u_{\eta}$ obtained in Lemma $4.3, \theta_{\eta}$ obtained in Lemma 4.4 and the damage $\xi_{\eta}$ obtained in Lemma 4.6 to construct the following Cauchy problem for the stress field. 
Problem $\mathbf{P V 4}_{\eta}$. Find a stress field $\boldsymbol{\sigma}_{\eta}:[0, T] \rightarrow \mathcal{H}$ such that

$$
\begin{aligned}
\boldsymbol{\sigma}_{\eta}(t) & =\mathcal{G}\left(\varepsilon\left(\mathbf{u}_{\eta}(t), \xi_{\eta}(t)\right)\right)+\int_{0}^{t} \mathcal{F}\left(\boldsymbol{\sigma}_{\eta}(s), \varepsilon\left(\mathbf{u}_{\eta}(s)\right)\right) d s-C_{e} \theta(t) \\
\forall t & \in[0, T] .
\end{aligned}
$$

In the study of Problem $\mathbf{P V 4}{ }_{\eta}$, we have the following result.

Lemma 4.7. $\mathbf{P V 4} 4_{\eta}$ has a unique solutions $\boldsymbol{\sigma}_{\eta} \in W^{1,2}(0, T ; \mathcal{H})$. Moreover, if $\boldsymbol{\sigma}_{i}, \mathbf{u}_{i}, \theta_{i}$ and $\xi_{i}$ represent the solutions of Problems $P V 4_{\eta}, P V 1_{\eta}, P V 2_{\eta}$ and, $P V 3_{\eta}$ respectively, for $\eta_{i} \in L^{2}\left(0, T ; V^{\prime}\right), i=1,2$ then there exists $C>0$ such that

$$
\begin{gathered}
\left|\boldsymbol{\sigma}_{1}(\mathbf{t})-\boldsymbol{\sigma}_{2}(\mathbf{t})\right|_{\mathcal{H}}^{2} \leq C\left(\left|\mathbf{u}_{1}(t)-\mathbf{u}_{2}(t)\right|_{V}^{2}+\left|\theta_{1}(t)-\theta_{2}(t)\right|_{L^{2}(\Omega)}^{2}+\right. \\
\left.\left|\xi_{1}(t)-\xi_{2}(t)\right|_{L^{2}(\Omega)}^{2}+\int_{0}^{T}\left|\mathbf{u}_{1}(s)-\mathbf{u}_{2}(s)\right|_{V}^{2} d s\right) \forall t \in[0, T]
\end{gathered}
$$

Proof. Let $\Lambda_{\eta}: L^{2}(0, T ; \mathcal{H}) \rightarrow L^{2}(0, T ; \mathcal{H})$ be the operator given by

$$
\Lambda_{\eta} \boldsymbol{\sigma}(\mathbf{t})=\mathcal{G}\left(\varepsilon\left(\mathbf{u}_{\eta}(t), \xi_{\eta}(t)\right)\right)+\int_{0}^{t} \mathcal{F}\left(\boldsymbol{\sigma}(s), \varepsilon\left(\mathbf{u}_{\eta}(s)\right)\right) d s-C_{e} \theta(t)
$$

for all $\boldsymbol{\sigma}_{\eta} \in L^{2}(0, T ; \mathcal{H})$ and $t \in[0, T]$. For $\boldsymbol{\sigma}_{1}, \boldsymbol{\sigma}_{2} \in L^{2}(0, T ; \mathcal{H})$, we use $(4.27)$ and (3.8) to obtain for all $t \in[0, T]$ :

$$
\left|\Lambda_{\eta} \boldsymbol{\sigma}_{1}(\mathbf{t})-\Lambda_{\eta} \boldsymbol{\sigma}_{2}(\mathbf{t})\right|_{\mathcal{H}} \leq L_{\mathcal{F}}\left|\boldsymbol{\sigma}_{1}(\mathbf{s})-\boldsymbol{\sigma}_{2}(\mathbf{s})\right|_{\mathcal{H}} d s
$$

It follows from this inequality that for large $p$ enough, the operator $\Lambda_{\eta}^{p}$ is a contraction on the Banach space $L^{2}(0, T ; \mathcal{H})$, and therefore there exists a unique element $\boldsymbol{\sigma}_{\eta} \in L^{2}(0, T ; \mathcal{H})$ such that $\Lambda_{\eta} \boldsymbol{\sigma}_{\eta}(\mathbf{t})=\boldsymbol{\sigma}_{\eta}$. Moreover, $\boldsymbol{\sigma}_{\eta}$ is the unique solution of Problem $\mathbf{P V} \mathbf{4}_{\eta}$, and using (4.25), the regularity of $\mathbf{u}_{\eta}$, the regularity of $\xi_{\eta}$, the regularity of $\theta_{\eta}$, and the properties of the operators $\mathcal{G}, \mathcal{F}$, and $C_{e}$, it follows that $\boldsymbol{\sigma}_{\eta} \in W^{1,2}\left(0, T ; V^{\prime}\right)$. Consider now $\boldsymbol{\eta}_{1}, \boldsymbol{\eta}_{2} \in L^{2}\left(0, T ; V^{\prime}\right)$ and for $i=1,2$ denote $\mathbf{u}_{\eta_{i}}=\mathbf{u}_{i}, \sigma_{\eta_{i}}=\sigma_{i}, \xi_{\eta_{i}}=\xi_{i}$ and $\theta_{\eta_{i}}=\theta_{i}$. We have

$$
\boldsymbol{\sigma}_{i}(\mathbf{t})=\mathcal{G}\left(\varepsilon\left(\mathbf{u}_{i}(t), \xi_{i}(t)\right)\right)+\int_{0}^{t} \mathcal{F}\left(\boldsymbol{\sigma}_{i}(s), \varepsilon\left(\mathbf{u}_{i}(s)\right)\right) d s-C_{e} \theta_{i}(t)
$$

and using the properties $(3.7),(3.8),(3.10)$ and of $\mathcal{G}, \mathcal{F}$ and $C_{e}$ we find

$$
\begin{aligned}
& \left|\boldsymbol{\sigma}_{1}(\mathbf{t})-\boldsymbol{\sigma}_{2}(\mathbf{t})\right|_{\mathcal{H}}^{2} \leq C\left(\left|\mathbf{u}_{1}(t)-\mathbf{u}_{2}(t)\right|_{V}^{2}+\left|\theta_{1}(t)-\theta_{2}(t)\right|_{L^{2}(\Omega)}^{2}+\left|\xi_{1}(t)-\xi_{2}(t)\right|_{L^{2}(\Omega)}^{2}\right. \\
& \left.+\int_{0}^{T}\left|\boldsymbol{\sigma}_{1}(\mathbf{s})-\boldsymbol{\sigma}_{2}(\mathbf{s})\right|_{\mathcal{H}}^{2} d s\right)+\int_{0}^{T}\left|\mathbf{u}_{1}(s)-\mathbf{u}_{2}(s)\right|_{V}^{2} d s, \quad \forall t \in[0, T] .
\end{aligned}
$$

We use Gronwall argument in the previous inequality to deduce (4.26), which concludes the proof of Lemma 4.7.

Finally, we define the operator

$$
\Lambda: L^{2}\left(0, T ; V^{\prime}\right) \rightarrow L^{2}\left(0, T ; V^{\prime}\right)
$$

by

$$
\begin{aligned}
& \left.(\Lambda \eta(\mathbf{t}), w)_{V^{\prime} \times V}=\left(\mathcal{G} \varepsilon\left(\mathbf{u}_{\eta}(t), \xi_{\eta}(t)\right)\right), \varepsilon(w)\right)_{\mathcal{H}} \\
& +\left(\int_{0}^{t} \mathcal{F}\left(\boldsymbol{\sigma}_{\eta}(s), \varepsilon\left(\mathbf{u}_{\eta}(s)\right)\right) d s-C_{e} \theta_{\eta}(t), \varepsilon(w)\right)_{\mathcal{H}}, \quad \forall t \in[0, T]
\end{aligned}
$$


Here, for every $\boldsymbol{\eta} \in L^{2}\left(0, T ; V^{\prime}\right) \mathbf{u}_{\eta}, \theta_{\eta}, \xi_{\eta}$ and $\sigma_{\eta}$ represent the displacement field, the temperature field, the damage and the stress field obtained in Lemmas 4.3, 4.4, 4.6 and 4.7 respectively. We have the following result.

Lemma 4.8. The operator $\Lambda$ has a unique fixed point $\boldsymbol{\eta} \in L^{2}\left(0, T ; V^{\prime}\right)$ such that $\Lambda \eta=\eta$.

Proof. Let now $\boldsymbol{\eta}_{1}, \boldsymbol{\eta}_{2} \in L^{2}\left(0, T ; V^{\prime}\right)$. We use the notation that $\mathbf{u}_{\eta_{i}}=\mathbf{u}_{i}, \dot{\mathbf{u}}_{\eta_{i}}=\mathbf{v}_{\eta_{i}}=$ $\mathbf{v}_{i}, \sigma_{\eta_{i}}=\sigma_{i}, \xi_{\eta_{i}}=\xi_{i}$ and $\theta_{\eta_{i}}=\theta_{i}$, for $i=1,2$. Using (3.4),(3.6),(3.8), (3.15), and (4.28) to find

$$
\begin{aligned}
& \left|\Lambda \boldsymbol{\eta}_{1}(\mathbf{t})-\Lambda \boldsymbol{\eta}_{2}(\mathbf{t})\right|_{V^{\prime}}^{2} \leq C\left(\left|\mathbf{u}_{1}(t)-\mathbf{u}_{2}(t)\right|_{V}^{2}+\left|\theta_{1}(t)-\theta_{2}(t)\right|_{L^{2}(\Omega)}^{2}+\left|\xi_{1}(t)-\xi_{2}(t)\right|_{L^{2}(\Omega)}^{2}\right. \\
& \left.+\int_{0}^{T}\left|\boldsymbol{\sigma}_{1}(\mathbf{s})-\boldsymbol{\sigma}_{2}(\mathbf{s})\right|_{\mathcal{H}}^{2} d s+\int_{0}^{T}\left|\mathbf{u}_{1}(s)-\mathbf{u}_{2}(s)\right|_{V}^{2} d s\right)
\end{aligned}
$$

We use the estimate (4.26) to obtain

$$
\begin{aligned}
& \left|\Lambda \boldsymbol{\eta}_{1}(\mathbf{t})-\Lambda \boldsymbol{\eta}_{2}(\mathbf{t})\right|_{V \prime}^{2} \leq C\left(\left|\mathbf{u}_{1}(t)-\mathbf{u}_{2}(t)\right|_{V}^{2}+\left|\theta_{1}(t)-\theta_{2}(t)\right|_{L^{2}(\Omega)}^{2}+\left|\xi_{1}(t)-\xi_{2}(t)\right|_{L^{2}(\Omega)}^{2}\right. \\
& \left.+\int_{0}^{T}\left|\mathbf{u}_{1}(s)-\mathbf{u}_{2}(s)\right|_{V}^{2}+\int_{0}^{T}\left|\theta_{1}(s)-\theta_{2}(s)\right|_{L^{2}(\Omega)}^{2} d s\right)
\end{aligned}
$$

Moreover, from (4.10) we obtain

$$
\begin{aligned}
& \left(\dot{\mathbf{v}}_{1}-\dot{\mathbf{v}}_{2}, \mathbf{v}_{1}-\mathbf{v}_{2}\right)_{V^{\prime} \times V}+\left(\mathcal{A} \mathbf{v}_{1}-\mathcal{A} \mathbf{v}_{2}, \mathbf{v}_{1}-\mathbf{v}_{2}\right)_{V^{\prime} \times V} \\
& \leq-\left(\eta_{1}-\eta_{2}, \mathbf{v}_{1}-\mathbf{v}_{2}\right)_{V^{\prime} \times V}
\end{aligned}
$$

We integrate this equality with respect to time.

We use the initial conditions $\mathbf{v}_{1}(0)=\mathbf{v}_{2}(0)=\mathbf{v}_{0}$, the relation (4.9) and (3.6) to find that

$$
m_{\mathcal{A}} \int_{0}^{T}\left|\mathbf{v}_{1}(s)-\mathbf{v}_{2}(s)\right|_{V}^{2} d s \leq C \int_{0}^{T}\left|\boldsymbol{\eta}_{1}(\mathbf{t})-\boldsymbol{\eta}_{2}(\mathbf{t})\right|_{V}\left|\mathbf{v}_{1}(s)-\mathbf{v}_{2}(s)\right|_{V} d s
$$

For all $t \in[0, T]$. Then, using the inequality $2 a b \leq \frac{a^{2}}{m_{\mathcal{A}}}+m_{\mathcal{A}} b^{2}$ we obtain

$$
\int_{0}^{T}\left|\mathbf{v}_{1}(s)-\mathbf{v}_{2}(s)\right|_{V}^{2} d s \leq C \int_{0}^{T}\left|\boldsymbol{\eta}_{1}(\mathbf{s})-\boldsymbol{\eta}_{2}(\mathbf{s})\right|_{V} d s, \quad \forall t \in[0, T]
$$

Since $\mathbf{u}_{1}(0)=\mathbf{u}_{2}(0)=\mathbf{u}_{0}$ we have

$$
\left|\mathbf{u}_{1}(t)-\mathbf{u}_{2}(t)\right|_{V}^{2} \leq C \int_{0}^{T}\left|\mathbf{v}_{1}(s)-\mathbf{v}_{2}(s)\right|_{V}^{2} d s
$$

We use the previous inequality and (4.30) to obtain

$$
\begin{gathered}
\left|\Lambda \boldsymbol{\eta}_{1}(\mathbf{t})-\Lambda \boldsymbol{\eta}_{2}(\mathbf{t})\right|_{V^{\prime}}^{2} \leq C\left(\int_{0}^{T}\left|\mathbf{v}_{1}(s)-\mathbf{v}_{2}(s)\right|_{V}^{2} d s+\right. \\
\left.\left|\theta_{1}(t)-\theta_{2}(t)\right|_{L^{2}(\Omega)}^{2}+\left|\xi_{1}(t)-\xi_{2}(t)\right|_{L^{2}(\Omega)}^{2}+\int_{0}^{T}\left|\theta_{1}(s)-\theta_{2}(s)\right|_{L^{2}(\Omega)}^{2} d s\right)
\end{gathered}
$$

The estimates (4.31) and (4.21) imply that

$$
\left|\Lambda \boldsymbol{\eta}_{1}(\mathbf{t})-\Lambda \boldsymbol{\eta}_{2}(\mathbf{t})\right|_{V^{\prime}}^{2} \leq \int_{0}^{T} C\left|\boldsymbol{\eta}_{1}(\mathbf{s})-\boldsymbol{\eta}_{2}(\mathbf{s})\right|_{V^{\prime}}^{2} d s
$$


Reiterating this inequality $m$ times leads to

$$
\left|\Lambda^{m} \boldsymbol{\eta}_{1}-\Lambda^{m} \boldsymbol{\eta}_{2}\right|_{L^{2}\left(0, T ; V^{\prime}\right)}^{2} \leq \frac{C^{m} T^{m}}{m !}\left|\boldsymbol{\eta}_{1}-\boldsymbol{\eta}_{2}\right|_{L^{2}\left(0, T ; V^{\prime}\right)}^{2}
$$

For $m$ sufficiently large, $\Lambda^{m}$ is a contraction on the Banach space $L^{2}\left(0, T ; V^{\prime}\right)$, and so $\Lambda$ has a unique fixed point.

Now, we have all the ingredients needed to prove Theorem 4.1.

Proof. Let $\boldsymbol{\eta}^{*} \in L^{2}\left(0, T ; V^{\prime}\right)$ be the fixed point of $\Lambda$ defined by (4.28) and denote

$$
\begin{gathered}
\mathbf{u}=\mathbf{u}_{\boldsymbol{\eta}^{*}}, \theta=\theta_{\boldsymbol{\eta}^{*}}, \xi=\xi_{\boldsymbol{\eta}^{*}}, \sigma=\sigma_{\boldsymbol{\eta}^{*}} \\
\boldsymbol{\sigma}=\mathcal{A} \varepsilon(\dot{\mathbf{u}})+\boldsymbol{\sigma}^{*}
\end{gathered}
$$

We prove that $(\mathbf{u}, \sigma, \xi, \theta)$, satisfies (3.24)-(3.28) and (4.1)-(4.4). Indeed, we write (4.25) for $\boldsymbol{\eta}=\boldsymbol{\eta}^{*}$ and use (4.32)-(4.33), we obtain that (3.24) is satisfied. We consider (4.5) for $\boldsymbol{\eta}=\boldsymbol{\eta}^{*}$ and use the first equality in (4.32) to find

$$
\begin{aligned}
& (\ddot{\mathbf{u}}(t), w-\dot{\mathbf{u}}(t))_{V^{\prime} \times V}+\left(\mathcal{A} \varepsilon(\dot{\mathbf{u}}), \varepsilon(w-\dot{\mathbf{u}}(\mathbf{t}))_{\mathcal{H}}+j(w)-j(\dot{\mathbf{u}}(t))\right. \\
& +\left(\eta^{*}(t), w-\dot{\mathbf{u}}(\mathbf{t})\right)_{V^{\prime} \times V} \geq(\mathbf{f}(t), w-\dot{\mathbf{u}}(t))_{V^{\prime} \times V}, \quad \forall w \in V
\end{aligned}
$$

Equation $\Lambda \boldsymbol{\eta}^{*}=\boldsymbol{\eta}^{*}$ combined with (4.28), (4.32) and (4.33) shows that

$$
\begin{aligned}
& \left(\eta^{*}(t), w\right)_{V^{\prime} \times V}=\mathcal{G}(\boldsymbol{\varepsilon}(\mathbf{u}(t)), \boldsymbol{\varepsilon}(w))_{\mathcal{H}}+ \\
& \left(\int_{0}^{t} \mathcal{F}(\boldsymbol{\sigma}(s)-\mathcal{A} \varepsilon(\dot{\mathbf{u}}(s)), \boldsymbol{\varepsilon}(\mathbf{u}(s))) d s-C_{e} \theta(t), \boldsymbol{\varepsilon}(w)\right) \quad \forall w \in V
\end{aligned}
$$

We now substitute (4.35) into (4.34) and use (4.33) to see that (3.25) is satisfied. We write (4.18) for $\boldsymbol{\eta}=\boldsymbol{\eta}^{*}$ and use (4.32) to find that (3.26) is also satisfied. Next, (3.28) is satisfied when the regularities (4.1) and (4.4) follow from Lemmas 4.3 and 4.4. The regularity $\boldsymbol{\sigma} \in L^{2}(0, T ; \mathcal{H})$ follows from Lemmas 4.3 and 4.4 , the assumptions (3.6) and (4.33). Finally (3.25) implies that

$$
\operatorname{div} \boldsymbol{\sigma}+\mathbf{f}_{0}(t)=\rho \ddot{\mathbf{u}}(t) \quad \text { in } V^{\prime}, \quad \text { a.e.t } \in[0, T]
$$

and therefore by (3.13) and (3.15), we find $\operatorname{div} \boldsymbol{\sigma} \in L^{2}\left(0, T ; V^{\prime}\right)$. We deduce that the regularity (4.3) holds which concludes the existence part of Theorem 4.1. The uniqueness of Theorem 4.1 is a consequence of the uniqueness of the fixed point of the operator $\Lambda$ defined by (4.28) and the unique solvability of Problems $\mathbf{P V} \mathbf{1}_{\eta}, \mathbf{P V} \mathbf{2}_{\eta}$, $\mathbf{P V} \mathbf{3}_{\eta}$ and $\mathbf{P V 4 _ { \eta }}$.

\section{References}

[1] Aissaoui, A., Hemici, N., Bilateral contact problem with adhesion and damage, Electron. J. Qual. Theory Differ. Equ., (2014), no. 18, 1-16.

[2] Barbu, V., Optimal Control of Variational Inequalities, Pitman, Boston, 1984.

[3] Boukaroura, I., Djabi, S., Analysis of a quasistatic contact problem with wear and damage for thermo-viscoelastic materials, Malaya Journal of Matematik, 6(2018), no. 2, 299-309.

[4] Djabi, S., A monotony method in quasistatic processes for viscoplastic materials with internal state variables, Revue Roumaine de Maths. Pures et Appliquées, 42(1997), no. 5-6, 401-408. 
[5] Djabi, S., A monotony method in quasistatic processes for viscoplastic materials with $E=E(e(u), k)$, Mathematical Reports (SCM), 2(52)(2000), no. 1.

[6] Djabi, A., Merouani, A., Bilateral contact problem with friction and wear for an elasticviscoplastic materials with damage, Taiwanese J. Math., 2015.

[7] Djabi, S., Sofonea, M., A fixed point method in quasistatic rate-type viscoplasticity, Appl. Math. and Comp Sci. 3(1993), no. 2, 269-279.

[8] Duvaut, G., Lions, J.L., Les Inéquations en Mécanique et en Physique, (French), (The Inequalities in Mechanics and Physics), Springer, Berlin, 1976.

[9] Ireman, P., Klarbring, A., Strömberg, N., A model of damage coupled to wear, Int. J. Solids Structures, 40(2003), 2957-2974.

[10] Merouani, A., Djabi, S., A monotony method in quasistatic processes for viscoplastic materials, Stud. Univ. Babeş-Bolyai Math., 3(2008), no. 1.

[11] Merouani, A., Messelmi, F., Dynamic evolution of damage in elastic-thermo-viscoplastic materials, Electron. J. Differential Equations, (2010), no. 129, 1-15.

[12] Touzaline, A., Analysis of a viscoelastic unilateral and frictional contact problem with adhesion, Stud. Univ. Babeş-Bolyai Math., 8(2013), no. 2, 263-278.

Ilyas Boukaroura

Applied Mathematics Laboratory, Department of Mathematics

Ferhat Abbas-Setif 1 University

and

Department of Mathematics, Mohamed Elbachir Elibrahimi

Bordj Bou Arreridj University

e-mail: ilyas_boukaroura@yahoo.fr

Seddik Djabi

Applied Mathematics Laboratory, Department of Mathematics

Ferhat Abbas, Sétif 1 University

e-mail: seddikdjabi@univ-setif.dz 